\title{
Potencial de utilização de resíduos do beneficiamento da erva mate (Ilex paraguariensis) na produção de painéis de partículas
}

\author{
Potential for use of wastes of yerba mate (llex paraguariensis) processing \\ in the production os particle boards
}

\author{
Alexsandro Bayestorff Cunha ${ }^{1}$, Carolina Alves Carvalho ${ }^{1}$, Rodrigo Buss ${ }^{1}$, \\ Deyvis Borges Waltrick ${ }^{1}$, Ricardo Ritter de Souza Barnasky', \\ Reny Aldo Henne ${ }^{1}$ e Rodrigo Antunes
}

\begin{abstract}
Resumo
A llex paraguariensis St Hill. (erva-mate) é uma espécie florestal explorada na Região Sul do Brasil para produção de chás e chimarrão por meio do processamento de suas folhas. O resíduo é direcionado para queima em caldeiras em função de ser um material de baixo valor agregado. O objetivo do estudo foi verificar o potencial de utilização de resíduos do beneficiamento da erva-mate na produção de painéis de partículas de média densidade. Foram produzidos painéis com massa específica nominal de $0,750 \mathrm{~g} / \mathrm{cm}^{3}$, dimensões de $40 \times 40 \times 1,6 \mathrm{~cm}, 12 \%$ de resina ureia formaldeído, $1 \%$ de emulsão de parafina, distribuídos em cinco tratamentos diferenciados na composição do colchão: (T1): 100\% de partículas de pinus, (T2): 75\% de partículas de pinus:25\% de partículas de erva-mate, (T3): 50\%:50\%, (T4): $25 \%: 75 \%$ e (T5): $100 \%$ de partículas de erva-mate. $\mathrm{O}$ ciclo de prensagem utilizado foi de $160^{\circ} \mathrm{C}$ de temperatura, pressão de $40 \mathrm{kgf} / \mathrm{cm}^{2}$ durante 8 minutos. A determinação das propriedades tecnológicas dos painéis foi realizada de acordo com os procedimentos da ASTM D1037 (1993), DIN 52362 (DIN, 1982) e NBR 14810 (ABNT, 2013). Como resultados, observou-se que os painéis, classificados como de média densidade, apresentaram valores médios para estabilidade dimensional aumentados com a inclusão de partículas de erva-mate. Para módulo de elasticidade e ruptura a flexão estática, a razão de compactação não seguiu a tendência apresentada na literatura, em função da menor razão de esbeltez das partículas de erva-mate, enquanto que para tração perpendicular, a maior quantidade de extrativos da erva-mate não prejudicou os resultados. $O$ resíduo do processamento da erva-mate apresentou potencial para utilização em painéis quando misturados na proporção 50:50 com partículas de Pinus spp.
\end{abstract}

Palavras-chave: painéis de madeira, matéria-prima alternativa, erva mate.

\begin{abstract}
Ilex paraguariensis St Hill. (yerba mate) is a forest species explored in the southern Region of Brazil for the production of tea and mate by the processing of its leaves. The residue is directed for burning in boilers because it is a material of low added value. The objective of the study was to verify the potential use of residues of yerba mate processing in the production of medium density particleboard. The panels were produced with nominal specific mass of $0,750 \mathrm{~g} / \mathrm{cm}^{3}$, dimensions of $40 \times 40 \times 1,6 \mathrm{~cm}, 12 \%$ of urea formaldehyde resin, $1 \%$ of wax emulsion, distributed in five treatments, differentiated in the composition of the mattress: (T1): $100 \%$ of pine particles, (T2): $75 \%$ of pine particles: $25 \%$ of yerba mate particles, (T3): $50 \%: 50 \%$, (T4): $25 \%: 75 \%$ and (T5): $100 \%$ of yerba mate particles. The press cycle used was $160^{\circ} \mathrm{C}$ of temperature, pressure of $40 \mathrm{kgf} / \mathrm{cm}^{2}$ for 8 minutes. The determination of the technological properties of the panels was carried out according to the procedures of ASTM D1037 (1993), DIN 52362 (DIN, 1982) and NBR 14810 (2013). It was found that the panels, classified as being of medium density, showed mean values for dimensional stability increasing with the inclusion of yerba mate particles. For the modulus of elasticity and rupture the static bending, the compaction ratio did not follow the trend presented in the literature, due to the lower slenderness ratio of the yerba mate particles, whereas for perpendicular traction, the greater amount of extracts of the yerba mate did not impair from the results. Yerba mate processing residues showed potential for use in panels when mixed in the 50:50 ratios with Pinus spp.
\end{abstract}

Keywords: wood panels, alternative raw material, yerba mate.

\footnotetext{
1. Departamento de Engenharia Florestal, Universidade do Estado de Santa Catarina - UDESC. Lages, SC, Brasil.

* Autor correspondente: alexsandro.cunha@udesc.br
} 


\section{INTRODUÇÃO}

A Ilex paraguariensis St Hill., popularmente conhecida como erva-mate, é uma espécie de grande expressão na região sul do Brasil, devido à sua importância econômica e cultural. A espécie tem sua origem na América do Sul, ocorrendo naturalmente na Argentina, Brasil e Paraguai. No Brasil, se distribui pelos estados do Mato Grosso do Sul, São Paulo e se estende até o Rio Grande do Sul (LORENZI, 2002). A atividade de extrativismo é realizada em quatro estados brasileiros, correspondente a um total de 346.953 toneladas no ano de 2016. Destaca-se como o maior produtor nacional o estado do Paraná (86,4\%), seguido de Santa Catarina (8,3\%) e Rio Grande do Sul (3,5\%) (IBGE, 2016).

No processamento, são utilizadas apenas as folhas e pequenos ramos que, no final do processo geram produtos como o chá e o chimarrão (MACCARI JÚNIOR, 2005). O resíduo gerado no beneficiamento das folhas, geralmente, é direcionado para queima em caldeiras para geração de energia.

Trabalhos vêm sendo desenvolvidos com o propósito de agregar um maior valor ao resíduo como, por exemplo, a adição de resíduo da secagem e cancheamento de erva-mate como cobertura morta, somando maior produtividade das ervateiras (LOURENÇO et al., 2001), bem como, a utilização para a produção de carvão vegetal, visando a remoção de contaminantes orgânicos em meio aquoso (GONÇALVES et al. 2007). Além disso, há estudos investigando a utilização de resíduos industriais da produção de erva-mate na dieta de bovinos leiteiros, com intuito de melhorar a nutrição animal (CHAVES, 2014).

No entanto, acredita-se em um potencial ainda maior para o resíduo lignocelulósico proveniente do processamento das folhas e ramos da I. paraguarienis, como na produção de painéis de partículas de média densidade, onde poderia ser utilizado como mix, juntamente com as espécies tradicionalmente utilizadas pelo setor, de forma a melhorar as propriedades relacionadas à estabilidade dimensional.

Outros resíduos agroindustriais já foram estudados para a produção de painéis de partículas como o sabugo de milho, as cascas de arroz, café, amendoim, mamona, coco, o pseudocaule de bananeira, o caule da mandioca e o bagaço de cana (MENDES et al., 2010).

O crescimento da incorporação de produtos à base de materiais lignocelulósicos (resíduos agroindustriais e fibras vegetais) na produção de painéis pode ser explicado pela diminuição da oferta de madeiras nativas comerciais e de reflorestamento, além do acentuado aumento no preço.

Na produção de painéis de partículas de média densidade, pode ser utilizada qualquer espécie de madeira ou material que contenha fibras (ROQUE; VALENÇA, 1998), desde que proporcionem resistência mecânica satisfatória com massa específica pré-estabelecida (MELO et al., 2009). Um limitante em relação à matéria-prima, é a massa específica do material, que deve ser de moderada a baixa, em função de que quanto menor a massa específica, maior o volume a ser utilizado, maior a razão de compactação, maiores as propriedades de resistência em rigidez, no entanto, as propriedades de estabilidade dimensional são prejudicadas. Desta forma, busca-se encontrar matérias primas que melhorem a estabilidade, sem prejudicar as propriedades mecânicas e que atendam os parâmetros estabelecidos pelas normas de referência.

O objetivo do estudo foi verificar o potencial de utilização de resíduos do beneficiamento da erva - mate (I. paraguariensis) na produção de painéis de partículas de média densidade, por meio da avaliação das propriedades tecnológicas.

\section{MATERIAL E MÉTODOS}

\section{Matéria-prima}

Foram utilizadas partículas de Pinus spp (Pinus taeda e Pinus elliottii), resíduos do processamento da erva-mate na forma de palitos, resina ureia-formaldeído e emulsão de parafina. As partículas de pinus foram coletadas diretamente no processo produtivo de painéis de média densidade da Empresa Bonet Madeiras e Papéis Ltda. (Santa Cecília, Santa Catarina), após a passagem das toras descascadas pelo cepilhador, sendo necessário a realização do processo de secagem até $4 \%$ de umidade em estufa com circulação forçada de ar.

Os palitos de erva-mate foram fornecidos por uma ervateira situada em Canoinhas, Santa Catarina. O resíduo proveniente da separação da erva-mate em pó, talos e palitos, sendo os dois últimos prejudiciais ao sabor do chá, portanto, destinados à queima. O preparo dos palitos para a produção 
dos painéis envolveu a transformação em partículas com dimensões adequadas em um moinho de martelo, a classificação em peneiras vibratórias e a secagem em estufa.

A resina ureia formaldeído e a emulsão de parafina com teor de sólidos de 65,1\% e 59,35\%, respectivamente, foram fornecidas pela empresa de painéis.

\section{Delineamento experimental}

O delineamento inteiramente casualizado envolveu a produção de 15 painéis com massa específica nominal de $0,750 \mathrm{~g} / \mathrm{cm}^{3}$, dimensões de 40 x $40 \mathrm{~cm}$, espessura de $1,6 \mathrm{~cm}, 12 \%$ de resina ureia formaldeído, $1 \%$ de emulsão de parafina; distribuídos em cinco tratamentos, que foram diferenciados na composição do colchão, sendo: (T1): 100\% de partículas de pinus, (T2): 75\% de partículas de pinus: 25\% de partículas de erva-mate, (T3): 50\%:50\%, (T4): 25\%:75\% e (T5): 100\% de partículas de erva-mate.

\section{Produção dos painéis}

As partículas foram proporcionalmente pesadas, de acordo com o tratamento, e colocadas em uma encoladeira, esta constituída de um tambor giratório e de uma pistola que pulverizava a resina e a parafina em momentos diferenciados, com auxílio de um compressor.

O colchão formado de partículas, resina e parafina foi submetido a pré-prensagem a frio com pressão específica de $5 \mathrm{kgf} / \mathrm{cm}^{2}$ por 10 minutos e na sequência a prensagem a quente com temperatura de $160^{\circ} \mathrm{C}$ e pressão de $40 \mathrm{kgf} / \mathrm{cm}^{2}$ por 8 minutos.

\section{Ensaios tecnológicos}

A massa específica das partículas de pinus e erva-mate foi determinada de acordo com a NBR 11941 (ABNT, 2003). As propriedades físicas e a resistência à tração perpendicular dos painéis foram determinadas pela ASTM D1037 (ASTM, 1993), enquanto a flexão estática pela DIN 52362 (DIN, 1982). Já a resistência ao arrancamento de parafuso foi determinada pela NBR 14810 (ABNT, 2013). A razão de compactação foi calculada por meio da relação entre a massa específica média dos painéis e a massa específica da madeira ou erva-mate.

\section{Análise dos dados}

Os valores encontrados em cada uma das propriedades, foram inicialmente avaliados para a verificação dos pressupostos para a utilização da estatística paramétrica. A distribuição da normalidade dos dados foi avaliada por meio do teste de Shapiro Wilk, enquanto a homogeneidade foi verificada pela aplicação do teste de Bartlett. Com os pressupostos atendidos, cada variável resposta foi submetida à análise de variância. Decorrendo rejeição da hipótese de nulidade, realizou-se o teste de médias de Scott-Knott a 95\% de probabilidade.

Quando não havia o atendimento aos pressupostos iniciais, os dados da propriedade de interesse eram transformados e posteriormente reavaliados. Ainda assim, permanecendo o não atendimento, era aplicada a estatística não paramétrica por meio do teste de Kruskal Wallis.

Além da análise estatística tradicional, os valores médios de cada tratamento foram comparados com os parâmetros das normas de qualidade: NBR 14810 (ABNT, 2013), ANSI A208.1 (ANSI, 2009), CS 236-66 (CS, 1968) e EN 312-2 (EN, 2003).

\section{RESULTADOS E DISCUSSÃO}

\section{Propriedades físicas}

Na Tabela 1 estão apresentados os valores médios para massa específica dos painéis, onde pode ser observado que não houve diferença estatística e que os coeficientes de variação dentro dos tratamentos foram baixos, demonstrando homogeneidade no processo de formação do colchão e na prensagem. No entanto, verifica-se que todos os tratamentos apresentaram massa específica inferior a nominal, com exceção do tratamento T1, formado exclusivamente por partículas de pinus. A amplitude dos valores de massa específica dos tratamentos, em relação a nominal aumentou com o acréscimo das partículas de erva-mate, em função da menor massa específica desta espécie $\left(0,354 \mathrm{~g} / \mathrm{cm}^{3}\right)$, o que, ao final da prensagem, acaba por aumentar a espessura, devido ao alívio da pressão e, também, segundo Eleotério (2000), a espalhar a massa para uma área maior que a planejada para o painel. 
Como a variação de massa específica foi de 0,705 a $0,757 \mathrm{~g} / \mathrm{cm}^{3}$, todos os painéis foram classificados como de média massa específica, conforme as normas CS 236-66 (CS, 1968), ANSI A208.1 (ANSI, 2009), NBR 14810 (2013) e EN 312-2 (EN, 2003).

Na razão de compactação foi constatada diferença estatística entre os tratamentos, onde os painéis compostos com maiores proporções de partículas de erva-mate apresentaram os maiores valores médios. Este fato é devido a menor massa específica da erva-mate, que na formação do colchão, acaba por ter um maior volume a ser compactado para uma mesma espessura de painel.

A relação inversamente proporcional entre a massa específica da matéria-prima e a razão de compactação também foi demonstrada por autores como Iwakiri et al. (2012) em pesquisa sobre espécies tropicais de diferentes massas específicas, Cunha et al. (2014) pesquisando a utilização de partículas de espécies de eucalipto com diferentes massas específicas e Trianoski et al. (2013) estudando a adição de Criptomeria japonica a painéis de pinus.

Um ponto importante a ser destacado na razão de compactação é a obtenção de valores acima do proposto por Maloney (1993) e Moslemi (1974) de forma a assegurar uma boa eficiência no processo de densificação e consolidação do painel até a sua espessura final. Assim, pode-se dizer que os valores de razão encontrados tendem a promover melhores coeficientes das propriedades mecânicas e piores no que tange a estabilidade dimensional dos painéis.

O valor médio de teor de umidade após a estabilização da massa foi de 8,98\%, com diferença estatística entre os tratamentos. Observa-se na Tabela 1, que os maiores teores de umidade foram encontrados nos painéis com maior proporção de partículas de erva-mate, o que é devido a maior quantidade de espaços disponíveis para a entrada das moléculas de água.

Tabela 1. Valores médios para massa específica, razão de compactação e teor de umidade.

Table 1. Mean values for specific gravity, compaction ratio and moisture.

\begin{tabular}{llcll}
\hline \multicolumn{1}{c}{ Tratamento } & MEP $\left(\mathbf{k g} / \mathbf{m}^{3}\right)$ & MEM $\left(\mathbf{k g} / \mathbf{m}^{\mathbf{3}}\right)$ & RC & TU (\%) \\
\hline $1\left(\mathrm{P}_{100} \mathrm{EM}_{00}\right)$ & $0,757 \mathrm{a}_{(4,53)}$ & 0,460 & $1,646 \mathrm{c}_{(4,53)}$ & $8,55 \mathrm{a}_{(10,50)}$ \\
$2\left(\mathrm{P}_{75} \mathrm{EM}_{25}\right)$ & $0,739 \mathrm{a}_{(6,57)}$ & 0,434 & $1,703 \mathrm{c}_{(6,57)}$ & $8,61 \mathrm{a}_{(13,78)}$ \\
$3\left(\mathrm{P}_{50} \mathrm{EM}_{50}\right)$ & $0,738 \mathrm{a}_{(6,42)}$ & 0,407 & $1,813 \mathrm{~b}_{(6,42)}$ & $8,70 \mathrm{a}_{(13,33)}$ \\
$4\left(\mathrm{P}_{25} \mathrm{EM}_{75}\right)$ & $0,713 \mathrm{a}_{(5,41)}$ & 0,381 & $1,871 \mathrm{a}_{(5,41)}$ & $9,22 \mathrm{~b}_{(13,99)}$ \\
$5\left(\mathrm{P}_{0} \mathrm{EM}_{100}\right)$ & $0,705 \mathrm{a}_{(4,69)}$ & 0,354 & $1,992 \mathrm{a}_{(4,69)}$ & $\left.9,82 \mathrm{~b}_{(12,12}\right)$ \\
Média & $\mathbf{0 , 7 3 0}$ & $\mathbf{0 , 4 0 7}$ & $\mathbf{1 , 8 0 5}$ & $\mathbf{8 , 9 8}$
\end{tabular}

ME Nominal $\quad 0,750$

Moslemi (1974) e Maloney (1993) Acima de 1,3

NBR 14810 (2013)

ANSI A208.1 (2009)

5 e $13 \%$

máximo $10 \%$

Legenda: MEP: massa específica dos painéis; MEM: massa específica da matéria-prima (madeira e erva-mate); RC: razão de compactação; TU: teor de umidade. Médias seguidas de mesma letra na coluna não diferem estatisticamente entre si pelo teste de Scott-Knott a $95 \%$ de probabilidade. Valores entre parênteses indicam o coeficiente de variação (\%).

Salienta-se que os valores encontrados para umidade estão abaixo da umidade de equilíbrio da sala de climatização que é de $12 \%$. A diminuição da higroscopicidade dos painéis está em função da redução da madeira em partículas e a posterior incorporação de resina, parafina e outros aditivos (WU, 1999) e, principalmente, às altas temperaturas aplicadas durante a etapa de prensagem, que fazem com que a pressão compacte as camadas externas do painel, tornando os sítios de adsorção menos disponíveis (DEL MENEZZI, 2004).

Ainda no teor de umidade dos painéis, nota-se que os valores médios estão de acordo com o especificado pela NBR 14810 (ABNT, 2013) e pela ANSI A208.1 (ANSI, 2009).

Para as propriedades relacionadas à estabilidade dimensional dos painéis, absorção de água e inchamento em espessura, Tabela 2, observa-se diferença significativa entres os painéis produzidos com quantidade de partículas de erva mate até $25 \%$ e os produzidos com porcentagem superior (acima de 50\%).

Desta forma, constata-se que a baixa massa específica da matéria-prima erva-mate $\left(0,354 \mathrm{~g} / \mathrm{cm}^{3}\right)$, juntamente com a menor massa específica dos painéis produzidos com a espécie, influenciaram diretamente nas propriedades supracitadas dos painéis, principalmente nos que continham mais de $25 \%$ de partículas, tendo em vista que os painéis possuem maior quantidades de espaços vazios 
Tabela 2. Valores médios para absorção de água e inchamento em espessura.

Table 2. Mean values for the water absorption and thickness swelling.

\begin{tabular}{|c|c|c|c|c|}
\hline \multirow{2}{*}{ Tratamento } & \multicolumn{2}{|c|}{ Absorção - AA (\%) } & \multicolumn{2}{|c|}{ Inchamento - IE (\%) } \\
\hline & $2 \mathrm{~h}$ & $24 \mathrm{~h}$ & $2 \mathrm{~h}$ & $24 \mathrm{~h}$ \\
\hline $1\left(P_{100} E_{00}\right)$ & $16,10 a_{(61,26)}$ & $40,44 \mathrm{a}_{(57,86)}$ & $10,85 \mathrm{a}_{(57,94)}$ & $18,45 a_{(26,46)}$ \\
\hline $2\left(P_{75} E_{25}\right)$ & $16,26 a_{(46,67)}$ & $51,88 \mathrm{a}_{(27,87)}$ & $11,48 a_{(13,13)}$ & $20,10 a_{(09,81)}$ \\
\hline $3\left(\mathrm{P}_{50} \mathrm{EM}_{50}\right)$ & $22,23 \mathrm{a}_{(48,42)}$ & $54,12 \mathrm{a}_{(24,45)}$ & $12,03 \mathrm{a}_{(20,08)}$ & $20,33 a_{(03,85)}$ \\
\hline $4\left(\mathrm{P}_{25} \mathrm{EM}_{75}\right)$ & $30,12 b_{(21,68)}$ & $58,77 b_{(10,81)}$ & $13,24 b_{(19,20)}$ & $23,00 b_{(08,81)}$ \\
\hline $5\left(P_{0} E M_{100}\right)$ & $32,33 b_{(29,86)}$ & $59,55 b_{(17,87)}$ & $14,51 b_{(16,78)}$ & $24,88 b_{(07,47)}$ \\
\hline Média & 23,40 & 52,95 & 12,42 & 21,35 \\
\hline NBR 14810 (2013) & & & & máx.18,00 \\
\hline ANSI A208.1 (2009) & & & & máx.40,00 \\
\hline CS 236-66 (1968) & & & & máx.35,00 \\
\hline EN 312-2 (2003) & & & & máx.15,00 \\
\hline
\end{tabular}

Médias seguidas de mesma letra na coluna não diferem estatisticamente entre si pelo teste de Scott-Knott a 95\% de probabilidade. Valores entre parênteses indicam o coeficiente de variação (\%).

disponíveis para a absorção da água; e a alta razão de compactação promove uma maior inchamento em espessura em função da maior quantidade de material por unidade de volume.

O fato da menor massa específica da matéria-prima e da alta razão de compactação produzir painéis com baixa estabilidade dimensional, ou seja, altos valores de absorção de água e inchamento em espessura, já é encontrado na maior parte dos trabalhos, como em painéis de partículas produzidos com coco, babaçu e Pinus spp. (MACHADO et al., 2017), capim-annoni e Pinus spp (BALDIN et al., 2016), casca de amendoim em composição com itaúba (BARBIRATO et al., 2014), além dos painéis produzidos exclusivamente com espécies florestais.

Comparando os resultados encontrados com outros trabalhos que estudaram resíduos agroindustriais na produção de painéis de partículas, tem-se Carvalho et al. (2015) que trabalharam com painéis de Pinus spp e resíduos da poda da erva-mate com e sem casca, encontrando valores entre 103\% e 124\% para AA 24 horas e entre 40\% e 46\% para IE 24 horas; Baldin et al. (2016), que utilizando capim-annoni, observaram valores para AA 24 horas entre 44\% e 149\% e para IE 24 horas, entre 31\% e 70\%. Da mesma forma, Machado et al. (2017) trabalhando com epicarpo e endocarpo de babaçu e Pinus spp, verificaram valores para AA 24 horas variando entre $70 \%$ e $80 \%$ e para IE 24 horas os valores ficaram entre 45\% e 51\%. Silva et al. (2015) trabalhando com bagaço de cana de açúcar e resíduos de Pinus spp (maravalha), encontraram valores para AA 24 horas variando entre 48 a 111\%, já para IE 24 horas os valores ficaram entre 10\% a 12. Igualmente, Barbirato et al. (2014) verificou valores para painéis com casca de amendoim em composição com itaúba para AA 24 horas entre $21 \%$ e $79 \%$ e para IE 24 horas entre de $6 \%$ a $24 \%$.

Quanto às normas de referência, que relacionam o IE 24 horas, verifica-se que os painéis de todos os tratamentos ficaram abaixo dos limites estabelecidos pelas normas ANSI A208.1 (ANSI, 2009) e CS 236-66 (CS, 1968), o que extremamente positivo; no entanto, ficaram acima do estabelecido pela NBR 14810 (ABNT, 2013) e EN 312-2 (EN, 2003), mesmo os painéis com 100\% de partículas de pinus, que é a espécie mais utilizada para a produção em nível comercial, fato que é recorrente em grande parte dos trabalhos com painéis reconstituídos de madeira.

\section{Propriedades mecânicas}

Os valores para módulo de elasticidade (MOE) e módulo de ruptura (MOR) a flexão estática, apresentados na Tabela 3, demonstram que houve diferença estatística entre os tratamentos para as duas propriedades, onde houve uma redução nos valores com o aumento da incorporação de partículas de erva-mate, no entanto, para MOR foi mais expressiva a partir de 75\%, e para MOE de 50\%.

A redução dos valores de MOE e MOR de forma gradativa com o aumento das partículas de erva-mate no painel, corrobora com o estudo de Carvalho et al. (2015), que encontraram, para painéis com Pinus spp (100\%), MOE médio de 1611,7 MPa e MOR de 19,6 MPa e, para painéis com erva-mate (100\%), 1180,1 MPa e 13,6 MPa. Entretanto, a diminuição dos valores de rigidez e resistência, com o acréscimo de matéria-prima de menor massa específica nos painéis e, consequentemente, maior razão de compactação dos mesmos, não é o que geralmente os estudos apontam quando se utiliza espécies florestais para a formação dos painéis, mas é o que tem se verificado quando se utiliza 
Tabela 3. Valores médios para flexão estática dos painéis.

Table 3. Mean values for the static bending of panels.

\begin{tabular}{lcc}
\hline \multicolumn{1}{c}{ Tratamento } & MOE (MPa) & MOR (MPa) \\
\hline $1\left(\mathrm{P}_{100} \mathrm{EM}_{00}\right)$ & $2232,41 \mathrm{a}_{(15,27)}$ & $20,42 \mathrm{a}_{(27,04)}$ \\
$2\left(\mathrm{P}_{75} \mathrm{EM}_{25}\right)$ & $2061,78 \mathrm{a}_{(15,43)}$ & $15,17 \mathrm{~b}_{(19,01)}$ \\
$3\left(\mathrm{P}_{50} \mathrm{EM}_{50}\right)$ & $1727,42 \mathrm{~b}_{(15,57)}$ & $12,52 \mathrm{c}_{(32,42)}$ \\
$4\left(\mathrm{P}_{25} \mathrm{EM}_{75}\right)$ & $1725,48 \mathrm{~b}_{(17,28)}$ & $9,91 \mathrm{c}_{(26,36)}$ \\
$5\left(\mathrm{P}_{0} E M_{100}\right)$ & $1442,50 \mathrm{c}_{(22,37)}$ & $9,31 \mathrm{c}_{(26,87)}$ \\
Média & 1837,92 & 13,47 \\
NBR 14810 (2013) & mín.1600 & mín. 11,00 \\
EN 312 (2003) & mín. 1600 & mín.13,00 \\
ANSI A208.1 (2009) & mín. 1550 & mín.10,00
\end{tabular}

Médias seguidas de mesma letra na coluna não diferem estatisticamente entre si pelo teste de Scott-Knott a 95\% de probabilidade. Valores entre parênteses indicam o coeficiente de variação (\%).

resíduos agrícolas / florestais, a exemplo dos estudos realizados por Baldin et al. (2016) com Pinus spp e capim annoni, Machado et al. (2017) com Pinus spp e babaçu, Melo et al. (2009), com Pinus spp e casca de arroz e Belini et al. (2010) com Eucalyptus grandis e bagaço da cana de açúcar.

Esta constatação é devido à geometria das partículas, ou seja, alta razão de esbeltez das partículas de Pinus spp e baixa da erva-mate. De acordo com Iwakiri (2005) e Vital et al. (1992), painéis produzidos com partículas com elevados índices de esbeltez, normalmente apresentam propriedades elevadas no ensaio de flexão estática, tanto que, no processo industrial, partículas maiores são destinadas para o centro do painel para conferir resistência e rigidez, e as menores para a superfície para proporcionar melhores características de acabamento e usinabilidade.

Em relação aos parâmetros das normas de referência para $\mathrm{MOE}$, todos os tratamentos se adequam, exceto o tratamento 5, que apresentou valor médio inferior ao exigido. Já para MOR, somente os tratamentos 1 e 2, com alta porcentagem de partículas de Pinus spp na sua composição, 100\% e $75 \%$, respectivamente, atenderam todas as normas. No entanto, o tratamento 3 (50:50\%), também atendeu a NBR 14810 (ABNT, 2013) e a ANSI A208.1 (ANSI, 2009).

Os valores médios para tração perpendicular e arrancamento do parafuso, tabela 4, indicam que os tratamentos foram equivalentes para a primeira propriedade, estando de acordo com os parâmetros das normas de referência. Já para o arrancamento do parafuso, mesmo apresentando valores superiores aos das normas, os tratamentos com maior quantidade de partículas de erva-mate, acima de 50\%, foram superiores.

Tabela 4. Valores médios para tração perpendicular e arranchamento do parafuso.

Table 4. Mean values for the perpendicular traction and screw withdrawal.

\begin{tabular}{|c|c|c|c|}
\hline \multirow{2}{*}{ Tratamento } & \multirow{2}{*}{$\begin{array}{l}\text { Tração Perpendicular } \\
\text { (Mpa) }\end{array}$} & \multicolumn{2}{|c|}{ Arrancamento do parafuso } \\
\hline & & Superfície (N) & Topo (N) \\
\hline $1\left(P_{100} E_{00}\right)$ & $0,40 a_{(26,27)}$ & $1790,04 \mathrm{~b}_{(26,42)}$ & $1493,13 \mathrm{a}_{(29,85)}$ \\
\hline $2\left(\mathrm{P}_{75} \mathrm{EM}_{25}\right)$ & $0,41 \mathrm{a}_{(15,86)}$ & $1804,25 b_{(18,04)}^{(20,4)}$ & $1514,08 \mathrm{a}_{(25,64)}$ \\
\hline $3\left(\mathrm{P}_{50} \mathrm{EM}_{50}\right)$ & $0,42 \mathrm{a}_{(09,97)}$ & $1943,04 \mathrm{a}_{(11,38)}$ & $1556,75 \mathrm{a}_{(23,52)}$ \\
\hline $4\left(\mathrm{P}_{25} \mathrm{EM}_{75}\right)$ & $0,42 a_{(11,37)}$ & $1952,79 a_{(17,06)}$ & $1560,58 \mathrm{a}_{(34,17)}$ \\
\hline $5\left(\mathrm{P}_{0} \mathrm{EM}_{100}\right)$ & $0,41 a_{(17,37)}$ & $1996,96 \mathrm{a}_{(18,90)}$ & $1587,04 \mathrm{a}_{(19,31)}$ \\
\hline Média & 0,41 & 1897,42 & 1542,32 \\
\hline NBR $14810(2013)$ & mín. 0,35 & mín. 1020 & mín. 800 \\
\hline EN 312 (2003) & mín. 0,35 & & \\
\hline ANSI A.208.1 (2009) & mín. 0,31 & mín. 800 & mín. 700 \\
\hline
\end{tabular}

Médias seguidas de mesma letra na coluna não diferem estatisticamente entre si pelo teste de Scott-Knott a 95\% de probabilidade. Valores entre parênteses indicam o coeficiente de variação (\%).

A não observância de diferença estatística entre os tratamentos para tração perpendicular, da mesma forma que Carvalho et al. (2015), não seguiu o que geralmente é encontrado na literatura, a exemplo de Moslemi (1974), que cita que quanto maior a razão de compactação e menor a razão de esbeltez, maiores são os valores para tração perpendicular, Haselein et al. (2002) que apontou que a resistência à tração perpendicular foi menor em painéis produzidos com partículas mais espessas e 
que a variável apresenta uma certa tendência de diminuição com a redução dos comprimentos das partículas, e Vital et al. (1992) que determinaram que a resistência a tração aumenta com a redução do comprimento da partícula. Assim, Brito e Peixoto (2000) justificam tal fato em função da melhor uniformização do material e pela menor quantidade de espaços vazios disponíveis.

A constatação observada no presente estudo pode ser decorrente da maior quantidade de extrativos presentes nas partículas de erva-mate em relação às de pinus, 6,01\% e 3,50\%, respectivamente, segundo Carvalho et al. (2015). Marra (1992) afirma que materiais lignocelulósicos com elevados teores de extrativos apresentam dificuldades de colagem, resultando em baixa resistência da ligação adesiva entre as partículas.

No entanto, este fato não impediu que todos os tratamentos atingissem os parâmetros mínimos exigidos pelas normas de qualidade, como foi o caso do estudo de Machado et al. (2017) com coco de babaçu e resina de mamona, Sartori et al. (2012) com bagaço da cana de açúcar e Varanda et al. (2015) com resíduos de aveia.

Contudo, em outros estudos, em que a matéria-prima apresentava elevada quantidade de extrativos, a resistência à tração perpendicular foi prejudicada de forma expressiva, como no estudo de Guimarães Júnior et al. (2016) com partículas de eucalipto e resíduos da cultura de sorgo, com extrativos de 5,04\% e 14,55\%, e Martins (2015) com eucalipto e vagem da soja, com 3,47\% e 26,72\%. Em ambos os estudos a razão de compactação era alta na presença de grande quantidade de resíduos agrícolas.

No arrancamento do parafuso, tanto superficial quanto de topo, foi verificado que as duas propriedades mecânicas foram as únicas a seguirem, numericamente, a tendência da razão de compactação, ou seja, aumentando com o acréscimo das partículas de erva-mate. No arrancamento de superfície a diferença estatística encontrada foi entre os tratamentos com maior quantidade de partículas de erva-mate (igual / acima de 50\%) e os com menor quantidade (igual / abaixo de 25\%), enquanto que para o topo não houve diferença, demonstrando boa uniformidade de distribuição das partículas na camada central do painel.

Os maiores valores médios encontrados para o arrancamento de superfície se justificam pela presença do gradiente de densidade formado durante o processo de prensagem e a localização do parafuso no ensaio de topo ser localizado exclusivamente no centro do painel, onde geralmente ocorre a menor densificação (TRIANOSKI et al., 2013).

Os resultados para arrancamento de parafuso estão de acordo com o encontrado por Carvalho et al. (2015), que encontraram 1742,6 N para painéis com Pinus spp e erva-mate, acima do determinado por Bianche et al. (2012) com Eucalyptus urophylla, Schizolobium paraiba e Sida spp, 1137,7 N, e Baldin et al. (2016) com Pinus spp. e Eragrostis plana (capim-annoni), 351,86 N.

\section{CONCLUSÃO}

Os painéis apresentaram aumento da razão de compactação com a inclusão gradativa de partículas de Ilex paraguariensis (erva-mate), o que refletiu na redução da estabilidade dimensional e no aumento da propriedade mecânica de arrancamento do parafuso.

Para flexão estática, o aumento da razão de compactação dos painéis com a inclusão de partículas de Ilex paraguariensis (erva-mate), não proporcionou, conforme literatura, aumento do módulo de elasticidade e de ruptura. Para tração perpendicular, não houve diferença estatística entre os tratamentos.

Os resíduos do processamento da Ilex paraguariensis (erva-mate) apresentaram potencial para utilização em painéis de partículas de média densidade, quando misturados na proporção 50:50 com partículas de Pinus spp. Para aumentar a proporção de partículas da matéria-prima mencionada, faz-se necessário um controle mais rigoroso no que tange a geometria das partículas.

\section{AGRADECIMENTOS}

Os autores agradecem à FAPESC - Fundação de Amparo à Pesquisa e Inovação do Estado de Santa Catarina, pelo financiamento concedido. 


\section{REFERÊNCIAS BIBLIOGRÁFICAS}

ABNT - ASSOCIAÇÃO BRASILEIRA DE NORMAS TÉCNICAS. NBR 14810: painéis de madeira de média densidade. Rio de Janeiro, 2013. 69 p.

ABNT - ASSOCIAÇÃO BRASILEIRA DE NORMAS TÉCNICAS. NBR 11941 - madeira: determinação da densidade básica. Rio de Janeiro, 2003. 6 p.

ANSI - AMERICAN NATIONAL STANDARDS INSTITUTE. ANSI A208.1 - Mat formed wood particleboard: specifications. Gaithersburg, 2009. 9 p.

ASTM - AMERICAN SOCIETY FOR TESTING AND MATERIALS. ASTM D1037: Standard test methods for evaluating properties of wood-base fiber and particle panel materials. Philadelphia, 1995. $32 \mathrm{p}$.

BALDIN, T.; SILVEIRA, A. G.; VIDRANO, B. R. A.; CANCIAN, L. C.; SPATT, L. L.; HASELEIN, C. R. Qualidade de painéis aglomerados produzidos com diferentes proporções de madeira e capim-annoni. Agrária, Recife, v. 11, p. 230-237, 2016.

BARBIRATO, G. H. A; FIORELlI, J.; GARZÓN, N.; PALLONE, E. A.; R; CRISTOFORO, A. L.; SAVASTANO JÚNIOR., H. Painel aglomerado híbrido de casca de amendoim reforçado com partículas de madeira itaúba. Ciência Florestal, v. 24, p. 685-697, 2014.

BELINI, U. L.; TOMAZELLO FILHO, M.; LOUZADA, J. L. P. C.; RODRIGUES, J. C. R. Aspectos anatômicos e tecnológicos de painéis confeccionados com fibras de eucalipto e cana-de-açúcar. Cerne, v.16, Suplemento, p. 48-52, 2010.

BIANCHE, J. J.; CARNEIRO, A. C. O.; VITAL, B. R.; PEREIRA, F. A.; SANTOS, R. C.; SORATTO, D. N. Propriedades de painéis aglomerados fabricados com partículas de eucalipto (Eucalyptus urophylla), paricá (Schizolobium amazonicum) e vassoura (Sida spp.). Cerne, v. 8, n.4, p. 623-630, 2012.

BRITO.E. O.; PEIXOTO, G. L. Avaliação da granulometria de partículas de Pinus taeda combinadas com adesivos comerciais para a fabricação de aglomerados. Floresta e Ambiente, Rio de Janeiro, v. 7, n. 1, p. 60-67, 2000.

CARVALHO, A. G.; ANDRADE, B. G.; CABRAL, C. P. T.; VITAL, B.R. Efeito da adição de resíduos de poda da erva-mate em painéis aglomerados. Árvore, v. 39, p. 209-214, 2015.

CHAVES, B.W. Utilização de resíduos industriais na dieta de bovinos leiteiros. Gestão, Educação e Tecnologia Ambiental, v. 18, p. 150-156, 2014.

CS - COMMERCIAL STANDART. CS 236-66: Mat formed wood particleboard. Geneva, 1968.

CUNHA, A. B.; LONGO, B. L.; RODRIGUES, A. A.; BREHMER, D. R. Produção de painéis de madeira aglomerada de Eucalyptus benthamii, Eucalyptus dunnii e Eucalyptus grandis. Scientia Forestalis, v.42, n.102, p.259-267, 2014.

DEL MENEZZI, C. H. S. Estabilização dimensional por meio do tratamento térmico e seus efeitos sobre as propriedades de painéis de partículas orientadas (OSB). 226 p. Tese (Doutorado em Ciências Florestais) - Universidade Federal do Paraná, Curitiba, 2004.

DIN - DEUTSCHES INSTITUT FÜR NORMUNG. Normen für Holzfaserplaten Spanplatten Sperrholz. DIN 52362: Testing of wood chipboards bending test, determination of bending strength. Berlin, 1982. 40 p.

EN - EUROPEAN STANDARD. EN 312: particleboards: specifications. London, 2003. 22p.

ELEOTÉRIO, J. R. Propriedades físicas de painéis MDF de diferentes densidades e teores de resina. 2000. 121 p. Dissertação (Mestrado Ciência e Tecnologia da Madeira) Escola Superior de Agricultura "Luís de Queiroz". Universidade de São Paulo, Piracicaba, 2000.

GONÇALVES, M.; GUERREIRO, M.C.; BIANCHI, M.L.; OLIVEIRA, L.C.A; PEREIRA, E.I; DALLAGO, R.M. Produção de carvão a partir de resíduo de Erva-Mate para a remoção de contaminantes orgânicos em meio aquoso. Ciência e Agrotecnologia, v. 31, p. 1386-1391, 2007.

GUIMARÃES JÚNIOR, J. B.; XAVIER, M. M.; SANTOS, T. S.; PROTASIO, T.P.; MENDES, R. F.; MENDES, L. M. Inclusão de resíduo da cultura de sorgo em painéis aglomerados de eucalipto. Pesquisa Florestal Brasileira, v. 36, p. 435-442, 2016.

HASELEIN, C.R.; CALEGARI, L.; BARROS, M.V.; HACK, C.; HILLIG, E.; PAULESKI, D.T.; POZZERA, F. Resistência mecânica e à umidade de painéis aglomerados com partículas de madeira de diferentes dimensões. Ciência Florestal, v. 12, n. 2, p. 127-134, 2002. 
Cunha et al. - Potencial de utilização de resíduos do beneficiamento da erva

mate (Ilex paraguariensis) na produção de painéis de partículas

IBGE - INSTITUTO BRASILEIRO DE GEOGRAFIA E ESTATÍSTICA. Produção da extração vegetal e silvicultura. Rio de Janeiro: IBGE, 2016. p.54 p.

IWAKIRI, S. Painéis de madeira reconstituída. Curitiba: FUPEF, 2005, 247 p.

IWAKIRI, S.; VIANEZ, B. F.; WEBER, C. TRIANOSKI, R.; ALMEIDA, V. C. Avaliação das propriedades de painéis aglomerados produzidos com resíduo de serrarias de nove espécies de madeiras tropicais da Amazônia. Acta Amazônica, Manaus, v.42, n.1, p.59-64, 2012.

LORENZI, H. Árvores Brasileiras: manual de identificação e cultivo de plantas arbóreas do Brasil. Nova Odessa: Plantarum, 2002. v. 1, 384 p.

LOURENÇO, R. S.; MEDRADO, M. J. S.; NIETSCHE, K.; SABATKE FILHO, F. E. Influência da cobertura morta na produtividade de erva-mate. Boletim de Pesquisa Florestal, n. 43, p. 113-122, 2001.

MACCARI JUNIOR, A. Análise do pré-processamento da erva-mate para chimarrão. 2005. 215 p. Tese (Doutorado em Engenharia Agrícola) - Faculdade de Engenharia Agrícola, Universidade Estadual de Campinas, Campinas, 2005.

MACHADO, N. A. F.; ANDRADE, H. A. F.; FURTADO, M. B.; SERRANO, L. J. P.; PARENTE, M. O. M.; MATOS, R. R. S. Propriedades físico-mecânicas de painéis multicamadas produzidas com partículas de coco babaçu e de Pinus sp. Agroambiente, v. 11, n. 3, p. 191-199, 2017.

MALONEY, T. M. Modern particleboard and dry-process fiberboard manufacturing. 2 ed. San Francisco: Miller Freeman, 1993. 689 p.

MARRA, A. A. Technology of wood bonding: principles and practice. New York, Van Nostrand Reinhold, $1992,453 \mathrm{p}$.

MARTINS, E. H. Aproveitamento do resíduo do processamento da soja para produção de painéis aglomerados. 2015. 43 p. Dissertação (Mestrado em Agronomia). Universidade Federal de Goiás, Jataí, 2015.

MELO, R. R.; SANTINI, E. J.; HASELEIN, C. R.; STANGERLIN, D. M. Propriedades físico-mecânicas de painéis aglomerados produzidos com diferentes proporções de madeira e casca de arroz. Ciência Florestal, v. 19, n. 4, p. 449-460, 2009.

MENDES, R. F.; MENDES, L. M.; GUIMARÃES JÚNIOR, J. B.; MORI, F. A.; CÉSAR, A. A. S. Efeito da incorporação de casca de café nas propriedades físico-mecânicas de painéis aglomerados de Eucalyptus urophylla S.T.Blake. Ciência e Agrotecnologia, v. 34, p. 610-617, 2010.

MOSLEMI, A. A. Particleboard. Illinois: Southern Illinois University, 1974. 244 p.

ROQUE, C. A. L.; VALENÇA, A. C. V. Painéis de Madeira Aglomerada. Rio de Janeiro: BNDES Setorial, 1998.19 p.

SARTORI, D. L.; CRAVO, J. C. M.; BARRERO, N. G.; FIORELLI, J.; SAVASTANO JÚNIOR, H. Painel em madeira de reflorestamento e chapas de partículas para instalações rurais. Floresta e Ambiente, v. 19, n. 2, p. 171-178, 2012.

SILVA, A. A.; BERGAMASCO R.; REZENDE, L. C. S. H.; SILVA, C. A.; FREITAS, B. O. APROVEITAMENTO DE RESÍDUOS DE MADEIRA DE PINUS E BAGAÇO DE CANA DE AÇÚCAR PARA PRODUÇÃO DE CHAPAS DE MADEIRA AGLOMERADO. Blucher Chemical Engineering Proceedings, v. 1, n. 2, p. 8399-8407, 2015.

TRIANOSKI, R.; IWAKIRI, S.; MATOS, J. L. M.; CHIES, D. Utilização da madeira de Cryptomeria japonica para produção de painéis aglomerados. Scientia Forestalis, v. 41, n. 97, p. 57-64, 2013.

VARANDA, L. D.; SOUZA, A. M.; POLETO, S. F. S.; SILVA, D. A. L.; PANZERA, T. H.; CHRISTOFORO, A. L.; ROCCO LAHR, F. A. Particleboards produced with agroindustrial wastes oat hulls (Avena sativa) and reflorestation wood. 1 ed. auru: Tiliform, v. 1, p. 249-276, 2015,

VITAL, B. R.; HASELEIN, C. R.; DELLA LUCIA, R. M. Efeito da geometria das partículas nas propriedades das chapas de madeira aglomerada de Eucalyptus grandis (Hill ex-Maiden). Árvore, v. 16, n. 1, p. 88-96,1992.

WU, Q. Application of Nelson's sorption isotherm to wood composities and overlays. Wood and Fiber Science, Pennsylvania, v. 31, n. 2, p. 187-191, 1999.

Recebido em: 15/05/2018

Aceito em: 25/03/2019 